\title{
The Unconstrained Binary Quadratic Programming Problem: A Survey
}

\author{
Gary Kochenberger \\ School of Business Administration, University of Colorado at Denver, Denver, CO 80217, \\ gary.kochenberger@cudenver.edu, Tel: 303-921-6372 \\ Jin-Kao Hao \\ LERIA, Université d'Angers, 2 Boulevard Lavoisier, 49045 \\ Angers, France, hao@info.iniv-angers.fr \\ Fred Glover \\ OptTek Inc \\ Boulder, Colorado 80302, glover@opttek.com \\ Mark Lewis \\ Craig School of Business, \\ Missouri Western State University, St Joseph, MO, 64507, mlewis14@missouriwestern.edu
}

Zhipeng Lü

School of Computer Science and Technology, Huazhong University of Science and Technology, 430074 Wuhan, China

Zhipeng.lui@gmail.com

Haibo Wang

Sanchez School of Business, Texas A\&M International University, Laredo, TX 78041, hwang@tamiu.edu

Yang Wang

LERIA, Université d'Angers, 2 Boulevard Lavoisier, 49045

Angers, France,yangw@info.iniv-angers.fr

\begin{abstract}
:
In recent years the unconstrained binary quadratic program (UBQP) has grown in importance in the field of combinatorial optimization due to its application potential and its computational challenge. Research on UBQP has generated a wide range of solution techniques for this basic model that encompasses a rich collection of problem types. In this paper we survey the literature on this important model, providing an overview of the applications and solution methods.
\end{abstract}

Keywords: Unconstrained binary quadratic programs, combinatorial optimization, metaheuristics 


\subsection{Introduction:}

The Unconstrained Binary Quadratic Programming (UBQP) problem is defined by

$$
\begin{aligned}
& \min x^{t} Q x \\
& \text { s.t. } x \in S
\end{aligned}
$$

where $\mathrm{S}$ represents the binary discrete set $\{0,1\}^{n}$ or $\{-1,1\}^{n}$ and $Q$ is an n-by-n square, symmetric matrix of coefficients. This simple model is notable for embracing a remarkable range of applications in combinatorial optimization. For example, the use of this model for representing and solving optimization problems on graphs, facility locations problems, resources allocation problems, clustering problems, set partitioning problems, various forms of assignment problems, sequencing and ordering problems, and many others have been reported in the literature.

Even more remarkable is the fact that, once given a UBQP formulation, these problems can be solved by a UBQP method which is not specialized to exploit the problem domain of any individual class of problems, to yield solutions whose quality in many cases rivals or even surpasses the quality of the solutions produced by the best specialized methods, while achieving this outcome with an efficiency that likewise rivals or surpasses the efficiency of leading specialized methods.

In this paper we survey the literature on UBQP, both its applications and solution methods. While many important constrained nonlinear binary models have been reported in the literature over the years, we focus our attention here on models that naturally occur in the form of an unconstrained quadratic binary model and those that have been re-cast into the form of UBQP. The paper is organized as follows. In section 2 we survey the range of applications that have been reported in the literature. Section 3 then presents a survey of the solution methodologies reported in the literature for solving UBQP. Section 4 highlights key theoretical work and this is followed by section 5 which wraps up the paper with our summary and conclusions. 


\subsection{Applications:}

Some reported applications appear naturally in the form of UBQP while others are "recast" into the UBQP form by employing various transformations. In the sub-sections below we examine these different categories of applications in turn. Within sub-sections, we present applications in the chronological order in which they appeared in the literature to give the reader a sense of when certain topics were addressed appeared in print, as well as progress made and trends in solution methodologies.

\subsection{Natural UBQP problems/applications}

The literature on UBQP goes back to the 1960s where the topics of pseudo-boolean functions and binary quadratic optimization were introduced by Hammer and Rudeanu (1968). Early papers related to UBQP concern applications in finance (Laughunn (1970)), project selection (Rhys(1970)), cluster analysis (Rao (1971)), economic analysis (Hammer and Shliffer (1971)), traffic management (Witzgall(1975)) and computer aided design (Krarup and Pruzan (1978)). While these applications actually take the form of constrained quadratic binary programs, they are mentioned here due to their historical role in fostering an interest in quadratic binary applications and also because several allow special cases that are precisely in the form of UBQP.

More recently many interesting applications that are expressed naturally in the form of UBQP have appeared in various papers. Barahona, Grotschell, Junger and Reinelt (1988) formulate and solve the problem of finding ground states of spin glasses with exterior magnetic fields, an important problem in physics, as an instance of UBQP. Computational results reveal that the model produces high quality solutions to spin glass problems of realistic size in reasonable amounts of computation time using 1980s technology.

Hansen and Jaumard (1990), in their work on the satisfiability problem, report their experience using the UBQP model as an approach for representing and solving small to medium sized Max 2-sat problems. Computational studies validated the attractiveness of this approach to the Max 2-sat problem in terms of quickly producing high quality solutions.

Boros and Hammer (1991) discuss the use of UBQP as an approach for modeling the Max-Cut problem. Their paper highlights the relationship between UBQP, Max-cut, Max 2-sat, and the Weighted Signed Graph Problem. The authors also present a discussion of valid 
inequalities and facets for polyhedra that provide the basis for further computational and theoretical work.

Alidaee, Kochenberger and Ahmadian (1994) discuss two machine scheduling problems in the context of UBQP: (1) scheduling $\mathrm{n}$ jobs on a single machine to minimize total weighted earliness and tardiness, and (2) scheduling $\mathrm{n}$ jobs on two parallel identical processors to minimize weighted mean flow time. In each case, the authors show how the problems can be modeled in a straight-forward manner as an instance of UBQP.

Pardalos and Xue (1994) indicate how the maximum clique problem can be modeled as an instance of UBQP. The authors also discuss the relationship between the maximum clique problem, the maximum independent set problem, and the vertex cover problem, indicating how each can be represented by UBQP. Finally, the authors provide a survey of solution methods for the maximum clique problem.

De Simone, Diehl, Junger, Mutzel, Reinelt, and Rinaldi (1995), as in the earlier 1988 paper by Barahona, Grotschell, Junger and Reinelt, adopt the UBQP model as a representation for the problem of finding ground states for the spin glass problem. In this 1995 paper the authors use the UBQP model to compute exact ground states for Ising spin glasses on 2dimensional grids with periodic boundary interactions, Gaussian bond distributions, and an exterior magnetic field. Preliminary experiments with a branch and cut algorithm for optimizing the UBQP form of the problem proved very promising, quickly producing high quality solutions to large spin glass instances.

Bomze, Budinich, Pardalos, and Pelillo (1999) discuss the maximum clique (MC) problem and how it can how it can be modeled in a variety of ways including a representation in terms of UBQP. The authors provide a very broad and in depth discussion of a variety of applications and of both exact and heuristic solution methods for the MC problem. Computational experience with various solution approaches to the MC problem is also presented.

Iasemidis, Pardalos, Sackellares, and Shiau (2001) discuss the use of the UBQP model as part of a process employed to predict the arrival of epileptic seizures. The entrainment between two brain sites can be quantified from measures of electrical activity (EEG) of the brain. The UBQP model was successful in identifying the most entrained sites leading to the optimal location of electrode sites. In clinical trials this procedure was successful in predicting epileptic seizures 20-40 minutes in advance of their occurrence. 
Alidaee, Glover, Kochenberger, and Rego (2005) discuss the number partitioning problem where the objective is to assign numbers to subsets such that the sums of the numbers in each subset are as close as possible to one another. The authors show that the $n=2$ subset case can be modeled as an instance of UBQP and that problems with $n>2$ can be modeled as a constrained version of UBQP. Extensions of the basic model along with computational experience for the $\mathrm{n}=2$ case are presented indicating the attractiveness of the approach.

Kochenberger, Glover, Alidaee, and Lewis (2005) discuss their experience with adopting the UBQP model to represent and solve max 2-sat problems. Expanding the computational scope reported earlier by Hansen and Jaumard (1990) on UBQP and the Max 2-sat problem, they offer extensive computational experience on very large test problems with up to 1000 variables and more than 10,000 clauses. Employing a basic form of tabu search to solve the UBQP instances, best known solutions to most test problems were found in a few seconds of computation time.

Neven, Rose and Macready (2008) discuss the use of quantum adiabatic algorithms, which represent new approaches to NP-hard combinatorial problems, for solving the image recognition problem. The authors indicate how the pattern recognition problem of deciding whether two images contain the same object can be modeled as an instance of UBQP, which they show is the general input format required by D-Wave superconducting quantum AQC processors. Computational experience was not reported.

Pajouh, Balasundaram, and Prokepyev (2013) discuss the use of the UBQP model for representing the maximal independent set problem. The authors present an analysis of local maxima properties along with relations between continuous local maxima of the quadratic formulation and the binary local maxima in the Hamming distance 1 and 2 neighborhoods. These results are then used to construct effective local search algorithms for the maximum independent set problem.

Kochenberger, Hao, Lu, Glover and Wang (2013) discuss the Max Cut problem and how the UBQP model can be effectively used to model and solve large scale instances. Using a tabu search algorithm, extensive computational testing is reported on problems with up to 10,000 variables. Comparisons with other solution methods from the literature for the max cut problem are provided, indicating the attractiveness of the UBQP/Tabu Search approach. 


\subsection{UBQP via reformulation}

The applications of the previous section illustrate the widespread usefulness of the UBQP model. The actual applicability of UBQP, however, is greatly extended due to re-formulation procedures that re-cast a constrained problem into an equivalent unconstrained binary quadratic model. Many re-formulations are accomplished by including quadratic infeasibility penalties in the objective function as an alternative to explicitly imposing constraints. In this manner a constrained model can be re-cast into the form of UBQP. In fact, any linear or quadratic problem with linear constraints and bounded integer variables can in principle be re-formulated as UBQP using quadratic penalties.

For several simple constraints, appropriate quadratic penalties are known in advance and can be used straight away. Examples of such penalties are given in Table1 below where $\mathrm{P}$ is a large positive scalar.

$$
\begin{array}{ll}
\text { Classical Constraint } & \text { Equivalent Penalty } \\
x+y \leq 1 & P(x y) \\
x+y \geq 1 & P(1-x-y+x y) \\
x+y=1 & P(1-x-y+2 x y) \\
x \leq y & P(x-x y) \\
x_{1}+x_{2}+x_{3} \leq 1 & P\left(x_{1} x_{2}+x_{1} x_{3}+x_{2} x_{3}\right)
\end{array}
$$

Table 1: Illustrative Known Penalties

Note that the penalty term in each case is zero if the associated constraint is satisfied, and otherwise the penalty is positive. These penalties, then, can be directly employed as an alternative to explicitly introducing the original constraints. For general constraints, however, appropriate penalty functions are not known in advance and need to be "discovered." A simple procedure (see for instance Hammer \& Rudeanu (1968); Hansen (1979); Hansen, Jaumard, \& Mathon (1993); and Boros \& Hammer( 2002) for finding an appropriate penalty for any linear constraint is given as follows: 
Consider the general constrained problem of the form

$$
\min x_{0}=x Q x
$$

$$
\text { s.t. } \quad A x=b, x \text { binary }
$$

This model accommodates both quadratic and linear objective functions since the linear case results when $\mathrm{Q}$ is a diagonal matrix (observing that $x_{\mathrm{j}}^{2}=x_{\mathrm{j}}$ when $x_{\mathrm{j}}$ is a $0-1$ variable). Under the assumption that $\mathrm{A}$ and $\mathrm{b}$ have integer components, problems with inequality constraints can also be put in this form by representing their bounded slack variables by a binary expansion. These constrained quadratic optimization models are converted into equivalent UQP models by adding a quadratic infeasibility penalty function to the objective function in place of explicitly imposing the constraints $A x=b$.

Specifically, for a positive scalar P:

$$
\begin{aligned}
x_{0} & =x Q x+P(A x-b)^{t}(A x-b) \\
& =x Q x+x D x+c \\
& =x \hat{Q} x+c
\end{aligned}
$$

where the matrix $\mathrm{D}$ and the additive constant $\mathrm{c}$ result directly from the matrix multiplication indicated. Dropping the additive constant, the equivalent unconstrained version of the constrained problem becomes

$$
U B Q P: \min x \hat{Q} x, x \text { binary }
$$

A suitable choice of the penalty scalar P can always be chosen so that the optimal solution to UBQP is the optimal solution to the original constrained problem. For ease of reference, the preceding procedure that transforms (1) into (2) will be called Transformation \# 1.

Transformation \#1 can be used in cases where an appropriate quadratic penalty function isn't known in advance. In certain special cases, as mentioned earlier, appropriate penalties are known and can be directly employed. One particularly important case that arises in many constrained combinatorial problems is: 
denoting a situation where a pair of binary choices are available and we must preclude choosing both. As shown in the preceding table, an equivalent quadratic penalty for this situation is simply

$$
P x_{j} x_{k}
$$

Due to the frequency with which the constraint of (3) appears in many important applications we single it out for special attention and refer to the penalty of (4) as an alternative to the constraint of (3) as Transformation \# 2. Many of the applications that follow were originally modeled as constrained 0/1 models and were recast into the form of UBQP by using Transformation 1 and/or 2 .

Other paths to reformulation exist as well. Often a well-chosen change of variable can result in transforming a constrained model into the form of UBQP. This is particularly important in the context of certain optimization problems on graphs where binary variables denoting whether or not an edge is chosen can be replaced by the product of the two associated binary node variables. In making such a substitution, we go from an "edge-oriented" model to a "nodeoriented" model. This typically results in a much smaller model in terms of both the number of variables and the number of constraints.

The clique partitioning problem affords a good example for illustrating this approach. The standard integer programming (IP) formulation (see for example Oosten, Rutten \& Spieksma (2001)) for clique partitioning is:

$$
\max \sum_{(i, j) \in E} w_{i j} x_{i j}
$$

s.t.

$$
\begin{aligned}
& x_{i j}+x_{i r}-x_{j r} \leq 1 \quad \forall \text { all distinct } i, j, r \in V \\
& x_{i j} \in\{0,1\} \text { for all }\{i, j\} \in E
\end{aligned}
$$

The variable $x_{i j}$ is equal to 1 if the edge $(\mathrm{i}, \mathrm{j})$ is in the partition and is equal to 0 otherwise. The coefficient $w_{i j}$ is the weight of the edge $(\mathrm{i}, \mathrm{j})$ in the graph. 
An alternative model results by changing from edge-based variables to node-based variables. For this new model we add artificial edges as needed to produce a complete graph and denote an upper bound on the number of cliques to be formed by Kmax. Then, letting $x_{i k}=1$ if node $\mathrm{i}$ is assigned to clique $\mathrm{k}$ and $x_{i k}=0$ otherwise, an equivalent model is:

$$
\begin{aligned}
& \max \sum_{i=1}^{n-1} \sum_{j=i+1}^{n} w_{i j} \sum_{k=1}^{K_{\max }} x_{i k} x_{j k} \\
& \text { s.t. } \\
& \sum_{k=1}^{k \max } x_{i k}=1 \quad \text { for } i=1, n
\end{aligned}
$$

In this formulation $n$ is the number of nodes in the graph and $w_{i j}$ again denotes the weight of edge $(\mathrm{i}, \mathrm{j})$. This model is much smaller than the standard IP in terms of both number of variables and the number of constraints. Note also that it is of the form:

$$
\begin{aligned}
& \max x^{\prime} Q x \\
& \text { s.t. } \\
& A x=b \quad x \text { binary }
\end{aligned}
$$

which can be re-cast into the form of UBQP using Transformation \#1.

\subsection{Specific Application Instances}

Each of the applications presented below were originally modeled as a constrained combinatorial problem and then re-cast into the form of UBQP. Once in this unified form the problems were successfully solved by various heuristic means.

Lewis, Alidaee, and Kochenberger (2005) address the problem of assigning tasks to processors in a distributed, multitasking computer architecture such that the sum of the resultant task completion costs and inter-task communication costs are minimized. The standard model for this problem is a constrained quadratic optimization model in binary variables with constraints ensuring that each task gets assigned to one of the processors available. The authors employ Transformation \# 1 to re-cast this model into the form of UBQP which in turn is solved with a basic tabu search heuristic. Computational experience with large-scale instances highlights the attractiveness of this approach. 
Kochenberger, Glover and Alidaee (2005) discuss the classic vertex coloring problem and how it can be effectively modeled and solved in the form of UBQP. A standard representation of the K-Coloring problem consists of two categories of constraints, one ensuring that each node gets a color, and the other ensuring that adjacent nodes receive different colors. The authors use Transformation \# 1 on the first set of constraints and Transformation \# 2 on the second to produce a UBQP representation of the problem. Computational experience applying a tabu search method to standard test problems from the literature indicates that this approach is very competitive with, and often superior to, specialized methods for vertex coloring.

Similar reformulations, using transformations \#1 and/or \#2 have been reported for other well-known combinatorial problems. Kochenberger, Glover, Alidaee, and Wang (2005) examine the use of UBQP as a tool for clustering microarray data into groups with high degrees of similarity. Wang, Alidaee, Glover, and Kochenberger (2006) discuss the problem of grouping machines and parts together in a flexible manufacturing system in a manner that facilitates economies in time and cost. Kochenberger, Alidaee, Glover, and Wang (2007) discuss the use of UBQP as a tool for modeling and solving the generalized independent set (GIS) problem. In each case the original model was re-cast into the form of UBQP and successfully solved in this new form.

In addition, Lewis, Kochenberger, and Alidaee (2008) discuss the classic set partitioning (SP) problem and how the UBQP framework can be utilized for modeling and solving this important class of problems. Computational experience using a basic tabu search heuristic on problems with up to 15,000 variables and 5,000 rows and various densities is presented with comparisons drawn with CPLEX. Also in 2008, Alidaee, Kochenberger, Lewis, Lewis, and Wang (2008) discuss the use of the UBQP model for representing and solving the well-known set packing problem. Favorable computational experience with a wide variety of set packing problems with up to 2,000 variables and 10,000 constraints is reported.

Lewis, Alidaee, Glover, and Kochenberger (2009) discuss the Linear Ordering (LO) problem and how it can be modeled and solved as an instance of UBQP. The standard model in the literature for LO is a large 0-1 linear program with many constraints. For instance, a model designed to order 150 items would have, in the classic model, more than one million constraints. Rather than use the general procedure of Transformation \# 1, the authors show how to easily re- 
cast the constrained model into the unconstrained form of UBQP by using a special quadratic penalty that is uniquely suitable for the problem at hand. Computational experience with both medium and large sized test problems reveals the effectiveness of this approach.

Douiri and Elbernoussi (2012) discuss the Sum Coloring Problem which generalizes the classical vertex coloring problem by seeking a valid coloring of vertices such that the sum of the colors assigned to all vertices in minimized. The transformation to UBQP is accomplished by using Transformation \# 1 on the constraints that ensure each vertex gets a color and Transformation \# 2 on the constraints that require adjacent nodes to have different colors. Computational experience with the resulting UBQP model was carried out using a genetic algorithm. Results obtained on a variety of standard test problems illustrate the attractiveness of this approach.

Wang and $\mathrm{Xu}$ (2013) discuss another variant of the classical vertex coloring problem, called the Robust Graph Coloring Problem (RGCP), where for a given feasible coloring, a penalty is incurred for each non-adjacent vertices that have the same color assigned. The optimization problem is to determine a feasible coloring that minimizes the sum of the penalties associated with the edges in the complementary edge set with endpoints that are assigned the same color. As with the previously discussed coloring problems, the transformation to UBQP is carried out using a combination of Transformation \#1 and Transformation \#2. Computational experience with several variations of a genetic algorithm on a set of test problems illustrates the effectiveness of the UBQP approach for modeling and solving RGCP.

Lewis, Kochenberger, Wang, and Glover (2013) discuss the use of UBQP for modeling and solving the Generalized Vertex Covering Problem (GVCP). GVCP generalizes the minimum weight vertex covering problem by employing a three tier cost structure for each edge and charging a cost depending on whether one, both or neither end point of a given edge is covered by the subset chosen. The optimization problem is to find the subset of nodes that minimizes the sum of both the node and edge costs. The model presented previously in the literature for GVCP is a large 0-1 linear program with a binary variable for each node in the graph and two binary variables for each edge. The authors here show how GVCP can readily be formulated as UBQP by employing a simple change of variable such that all edge variables and 
all constraints are eliminated. Computational experience comparing the original linear model and the equivalent UBQP model illustrates the superiority of UBQP for this class of problems.

\subsection{Solution Methods}

While a few special cases of UBQP are polynomially solvable (see for instance Picard (1976), Barahona (1986), Pardalos and Jha (1991)), UBQP in general is an NP-hard problem (see Pardalos and Jha (1992)) and for all but small to moderate sized problems, heuristic methods are required to produce good solutions in a reasonable amount of computer time. Nonetheless, there is a sizable literature on exact methods for UBQP. In the sub-sections below we first survey the exact methods that have appeared in the literature followed by the heuristic methods described in the literature for solving UBQP.

\subsection{Exact Methods}

The literature on exact methods for UBQP introduces a variety of algorithms, each with the virtue of terminating, given enough time and memory, with a globally optimal solution. Most approaches involve a tree search of a general branch-and-bound nature but other methods exist as well. In this section we survey, in chronological order, the prominent methods reported over the past thirty-plus years.

Gulati, Gupta, and Mittal (1984) describe a branch and prune algorithm for solving UBQP which is designed to determine all local minimizing points, terminating with the global optimal solution revealed as the incumbent. Computational experience with random test problems with up to 125 variables is given.

Carter (1984) proposes a branch and bound algorithm for UBQP that first employs modified form of Cholesky factorization to transform an indefinite instance of UBQP into an equivalent positive definite form of the problem. Variable elimination based on hessian information is used accelerate search process. Computational experience on a variety of random test problems with various characteristics and with up to 30 variables is given.

Williams (1985) describes a branch and bound algorithm for UBQP that successfully solved a set of randomly generated test problems with up to 100 variables. The algorithm begins with a reduction procedure that obtains a good starting solution and subsequently uses the "roof 
dual" to help guide the depth-first branch and bound search. Comparisons with other methods are given.

Barahona, Junger, and Reinelt (1989) describe an approach to solving UBQP that first reduces the problem to an equivalent instance of a max-cut problem. This, in turn, is solved by a linear programming-based branch and bound method. Constraints based on the cut polytope are used to improve node information and enhance the search process. Computational experience is reported on random problems up to size 100 variables along with comparisons with other methods.

Kalantari and Bagchi (1990) describe the adaptation of an algorithm for minimizing linearly constrained concave quadratic functions for the purpose of solving UBQP. Their method starts with a transformation to ensure the $\mathrm{Q}$ matrix is positive definite, giving an equivalent concave quadratic minimization problem. The authors then describe their branchand-bound method where subproblems are defined by fixing a variable at zero or one and bounds are computed by minimizing a linear convex envelope over the feasible region of the subproblem. Computational testing is reported on random problems with up to 50 variables.

Pardalos and Rodgers (1990a) describe a branch-and-bound algorithm for solving UBQP that successfully solved a variety of test problems with up to 200 variables. The algorithm, which uses no multiplications or divisions, incorporates dynamic preprocessing techniques for fixing variables and heuristics for finding good starting points. In (1990b) the authors describe a parallel version of the algorithm implemented and tested on a hypercube architecture. Computational experience and an analysis of the speedup achieved are presented. Then in (1992), the authors describe a variation of their branch-and-bound algorithm designed for the UBQP representation of the maximum clique problem. Extensive computational experience is reported for alternative branching rules and data structures, in route to producing a specialized algorithm optimized for the maximum clique problem with up to 1000 vertices and 150,000 edges.

Billionnet and Sutter (1994) describe a branch-and-bound algorithm for solving UBQP that successfully solved a large variety of random test problems with up to 100 variables. Their innovation was in the calculation of lower bounds to guide the search process. At each node in 
the search tree, a lower bound is computed by combining information obtained from roof duality, a quadratic posiform associated with the directed cycles of an implications graph, and a component obtained from the induced posiform of degree 4. Computational experience is given comparing the method with other methods.

Palubeckis (1995) describes a branch-and-bound algorithm for solving UBQP utilizing heuristically generated subproblem solutions that are mapped onto the zero n-vector leading to transformed subproblems in the form of the original UBQP model. Special classes of polytope facets are employed in computing bounds used to guide the search process. Computational experience with random problems with up to 100 variables, and additional experience with several real problems having to do with printed circuit board design, illustrate the efficiency of the method and indicate that it compares favorably with other contemporary methods.

Helmberg and Rendl (1998) describe a branch-and-bound algorithm for solving UBQP based on semidefinite relaxations and cutting planes to enhance the quality of the bounds produced. The semidefinite relaxations are solved by an interior point algorithm specialized for semi-definite programs. Computational experience is reported on a set of UBQP instances of the max-cut variety with up to 100 variables. While the approach was robust in that it was successful in solving the test problems attempted, run times were generally not competitive with other recently reported exact methods for UBQP.

Hansen, Jaumard, and Meyer (2000) describe an enhanced version of the branch-andbound method of Pardalos and Rodgers (1990a) that led to favorable comparisons with the original algorithm on a standard set of problems with up to 100 variables. The new method employs improved bounds obtained by first transforming the problem to an equivalent posiform which yields tighter roof dual bounds as well as effective variable elimination test that efficiently guide the tree search process. The roof dual bounds are computed via a maximum flow algorithm.

Huang, Pardalos, and Prokopyev (2006) describe a depth-first branch-and-bound method that begins by first formulating an equivalent bi-level formulation of UBQP. This new formulation facilitates bounding procedures and pruning strategies, utilizing a gradient midpoint 
method that proved to be effective in early testing. Computational experience with random test problems of various densities and with up to 60 variables is presented.

Pardalos, Prokopyev, and Busygin (2006) discuss the connections between discrete optimization and continuous optimization in general with a focus on formulations that embed the initial discrete domain into a larger continuous space. The authors then focus on the general UBQP model, indicating how a reformulation based on an appropriate diagonal perturbation, causing the Q matrix to be negative semidefinite, yields an equivalent continuous problem of minimizing a quadratic concave function over the unit hypercube. A discussion of this approach applied to the maximum clique problem is given.

Pan, Tan, and Jiang (2008) describe a continuous approach for solving UBQP based on the Fischer-Burmeister nonlinear complementarity function. Rather than employing relaxations and bounding information in a tree search scheme, the authors reformulate UBQP as a continuous problem with equilibrium constraints. In turn, the optimal solution to this model is found by a global continuation algorithm utilizing a strictly convex global smoothing function and solving a sequence of unconstrained minimization problems. Computational experience is reported with random problems with up to 1000 variables, indicating the effectiveness of this approach.

Gueye and Michelon (2009) present a general framework for constructing linearizations of UBQP which, in turn, can be solved in principle by standard optimizers for mixed integer linear programs. The framework, which contains existing linearization methods in the literature as special cases, consists of decomposing the objective function into component matrices, identifying a complete linear representation of the polytope for each component, and then adding constraints that link the components together. A new linearization, derived from the general framework, is described and computational comparisons are given with existing methods illustrating the potential of the new approach.

Dinh, Canh, and Thi (2010) discuss a new continuous approach for solving UBQP that is based on DC (difference of convex functions) programming. In their approach, principles of DC programming are used to develop a local optimization algorithm (DCA) that solves a finite number of linear programs leading to a locally optimal solution. Globally optimal solutions are 
produced by embedding DCA in a branch-and-bound algorithm. Computational experience on problems from the literature with up to 100 variables, along with comparison with other methods, is given.

Mauri and Lorena (2011) present a new algorithm for solving UBQP based on Largangean decompositions. Their method starts with a linearization of UBQP represented by a graph. In turn, the graph is partitioned into clusters of vertices forming a dual problem that is solved by a subgradient algorithm. Clusters are formed using the well-known METIS heuristic and the linear Lagrangean subproblems are solved using CPLEX. In (2012a) the authors present a column generation alternative to the subgradient algorithm leading to performance improvement. In (2012b) the authors present and test further enhancements to their column generations approach for solving UBQP. Throughout all, computational experience on standard UBQP test problems with up 500 variables is given. Comparisons with other decompositionbased methods are given indicating the potential of the procedures proposed here.

Li, Sun, and Liu (2012) present a new algorithm for solving UBQP based on the inherent geometric properties of the minimum circumscribed sphere containing the ellipsoidal contour of the objective function. Based on these properties, effective bounding information as well as new procedures for optimally fixing variables are derived. In addition, this geometric approach led to new optimality conditions for UBQP. The new bounding techniques and variable fixing conditions were combined in a branch-and-bound method and tested on standard problems with up to 200 variables. Comparisons drawn with other recent methods in the literature indicate the attractiveness of the method proposed.

In addition to the exact methods surveyed above from the literature, we point out that several commercial methods, based on branch-and-cut techniques, are now available and hold considerable promise for directly optimizing moderate sized instances of UBQP. See for example the paper by Billionnet \& Elloumi ( 2007) which reports on the use of the branch-and cut quadratic integer optimizer available from CPLEX.

\subsection{Heuristic and Metaheuristic Methods}

The NP-hard nature of UBQP along with its application potential has motivated a large number of papers in recent years describing various heuristic methods for quickly finding high 

are simple enough to qualify as heuristics, those that generate the best solutions are metaheuristic procedures that incorporate compound strategies considerably more advanced than in the basic heuristics. These methods are surveyed below:

Boros, Hammer and Sun (1989) develop a Devour Digest Tidy-up (DDT) procedure to rapidly obtain a solution to UBQP. Based on the posiform expression of UBQP's objective function, the proposed method includes devour, digest and tidy-up phases. The devour phase identifies a term with the largest coefficient and sets it to 0 in terms of minimization. The digest phase draws logical conclusions for the items from the devour phase. The tidy-up phase finally substitutes the logical consequences previously derived into the current quadratic function. Computational experience indicates the effectiveness of the method proposed, in particular on problems of low density.

Glover, Kochenberger, and Alidaee (1998) propose an adaptive memory tabu search algorithm, which incorporates a strategic oscillation scheme to enable the search to go beyond the local optimum obtained by constructive and destructive phases. A key feature of this method lies in the use of a critical event memory, that collects recency and frequency information from critical events (moves that causes the solution values to decrease), to guide the oscillation process. Another feature lies in the use of adaptive oscillation depths. Extensive computational experience discloses that the proposed method outperforms the best exact and heuristic methods previously reported in the literature in terms of speed and solution quality.

Glover, Kochenberger, Alidaee and Amini (1999) describe an enhanced version of their previous adaptive memory tabu search algorithm. A simple but effective scheme is proposed for accelerating the evaluation of moves and for updating associated problem information. In addition, methods for generating high quality initial solutions and for creating additional trial solutions at critical events are also introduced. Computational experience with up to 1000 variables reveals this enhanced version can produce high quality solutions within several minutes.

Beasley (1998) adapts tabu search and simulated annealing to solving UBQP. The tabu search implementation incorporates a strategy in which, once an improved solution is found, a 
simple local search is successively employed to perform moves irrespective of their tabu status. Contrary to the tabu search procedure, a local search is applied only at the end of the simulated annealing process. Computational comparisons indicate that their tabu search generally performs better than simulated annealing for small and medium instances but worse for large instances.

Alkhamis, Hasan, and Ahmed (1998) present a simulated annealing based heuristic with a well-selected cooling schedule. Tested on several hundred test problems, the proposed heuristic outperforms several algorithms based on bounding techniques, in particular with respect to computational time. Additional analysis shows that initial solutions and the matrix density have limited influence on the effectiveness of the simulated annealing algorithm.

Merz and Freisleben (1999) devise a hybrid genetic algorithm, in which a simple local search is incorporated into the traditional genetic algorithm. The crossover operator is a variant of uniform crossover, requiring the generated offspring solutions to have the same hamming distance from the parents. The population updating criterion refers to the quality of solutions, assuring that each solution occurs only once in the population, as customarily done in scatter search methods. A diversification component is launched when the average hamming distance of the population drops below a threshold or the population is not updated for more than 30 consecutive generations. Computational experience shows that a simple genetic algorithm is sufficient to find best known results for problem instances with less than 200 variables but for those with a large number of variables, local search is needed for attaining high quality solutions.

Amini, Alidaee, and Kochenberger (1999) present a scatter search approach, which consists of a diversification generation method, a solution improvement method, a reference set update method, a subset generation method and a solution combination method. The diversification generation method systematically generates a collection of diverse trial solutions based on a seed solution by setting an incremental parameter that determines which bits of the seed solution should be flipped. The improvement method performs a compound move that sequentially cycles among three types of candidate moves until no attractive move can be identified. The reference set update method replaces solutions in the reference set with new candidate solutions using the quality measurement. The solution combination method uses linear combination of solutions in a subset derived from the subset generation method to produce new solutions. Since some variables may receive fractional values in the solution obtained in the 
linear combination, a rounding procedure is employed to recover integer values. Experiments on three classes of problems show the attractiveness of the proposed method.

Lodi, Allemand, and Liebling (1999) present an evolutionary method for solving UBQP. The proposed algorithm is characterized by the following features. First, a preprocessing phase is applied to fix certain variables at their optimal values and keep them unchanged during each successive round of local search, hence resulting in a reduced problem scale. Second, a local search procedure that alternates between construction phase and destructive phases is used to get an improved solution. Finally, a uniform crossover operator is used to produce offspring solutions, where variables with common values in parental solutions are temporarily fixed in this round of local search. Computational experience on problem instances with up to 500 variables is given. A further analysis demonstrates that the preprocessing phase is effective for small problem instances but is unable to appreciably reduce the problem size for large ones.

Katayama, Tani, and Narihisa (2000) propose a genetic local search algorithm for solving UBQP. Their local search procedure integrates 1 -flip moves dedicated to going into new good search area and $k$-flip moves dedicated to solution improvement. A traditional uniform crossover and a simple mutation operator are joined to generate a suitable offspring solution. A diversification/restart strategy is incorporated to maintain a diversified population. Tests on large problem instances indicate the effectiveness of the proposed algorithm.

Katayama and Narihisa (2001) present a simulated annealing algorithm with an innovative use of multiple annealing processes to enhance the search. Each annealing process takes the best solution found in the previous annealing process as the initial solution and employs a different initial temperature. Experimental results demonstrate the performance of the proposed algorithm, especially for large instances with 2500 variables.

Merz and Freisleben (2002) describe a greedy heuristic and two local search algorithms based upon 1-flip and k-flip neighborhoods. The greedy construction procedure starts from a solution with all variables assigned to 0.5 (the so called third state) and each constructive step picks a variable with probability proportional to the gain value when changing the variable's value from 0.5 to 0 or 1 . Each iteration of the 1-flip local search proceeds to the neighbor solution with the best solution quality. The $k$-flip local search borrows the idea from the Lin- 
Kernighan algorithm of Kernighan and Lin (1972) for solving the graph partitioning problem to efficiently reduce the neighborhood exploration. Each $k$-flip move consists in repeating performing the best 1-flip move until all 1-flip moves are performed and picking the best from the resulting solutions. Computational comparisons disclose the superiority of the multistart $k$ flip local search combined with randomized greedy initial solutions.

Glover, Alidaee, Rego, and Kochenberger (2002) propose several one-pass heuristics to advance the Devour Digest Tidy-up (DDT) method of Boros, Hammer and Sun (1989). Based on the hypothesis that setting multiple variables with value 1 or 0 in a pass may lead to worse performance, the idea is to guarantee only one variable gets the implied assignment in each pass. The proposed one-pass heuristics differ in strategies for evaluating contributions of variables. Computational experience indicates that the method outperforms the DDT method but no single one-pass heuristic dominates the others on every problem instance.

Palubeckis and Tomkevicius (2002) present a greedy random adaptive search procedure (GRASP) which cycles between a construction phase and a local search phase. Each step in the construction phase picks a variable from a candidate list with probability proportional to the gain value of this variable, where the candidate list is composed of a certain number of variables with the largest gain values, calculated according to a specific gain function. The local search phase implements a simple ascent algorithm. Two enhanced versions are tested, which result by replacing local search with tabu search and by combining a classic random restarting procedure with tabu search. Computational comparisons illustrate the merit of incorporating greedy construction based initial solutions and tabu search.

Palubeckis (2004) examines five multistart tabu search strategies dedicated to the construction of an initial solution. The first multistart strategy produces a new initial solution in a random way. The second identifies a candidate set of variables whose values are prone to change when moving from the current solution to an optimal one and then applies a steepest ascent algorithm where variables not included in this candidate set are fixed at specific values. The third multistart strategy is the same as the constructive phase proposed in Palubeckis, 2002. The fourth uses a set of elite solutions to calculate the probability of each variable being assigned value 1 . If the probability for a given variable is larger than 0.5 , then this variable is assigned to be 1 in the constructed solution; otherwise it is assigned to be 0 . The last multistart strategy uses 

search on the modified instance. Extensive comparisons on problem instances with up to 7000 variables demonstrate the algorithm using the second multistart strategy performs better than the other proposed alternatives.

Merz and Katayama (2004) conduct landscape analysis and observe that (1) local optima of the UBQP problem instances are concentrated in a small fraction of the search space; (2) the fitness of local optima and the distance between local optima and the global optimum are correlated. Based on the observations, they propose a memetic algorithm in which an innovative variation operator is used to generate an offspring solution and the $k$-flip local search proposed in Katayama, Tani, and Narihisa (2000) is used to improve solution quality. The variation operator introduces new alleles not contained in both parents by referring to the move gain of performing 1-flip moves, avoiding the rediscovery of local optima already extensively visited. Comparisons with other algorithms demonstrate the effectiveness of the proposed algorithm.

Boros, Hammer, and Tavares (2006) present several preprocessing techniques to simplify the UBQP problem. The purpose of the preprocessing simplification is to provide several features, including lower bounds for the minimum of the objective function, optimal assignments for some variables, and binary relations between the values of certain pairs of variables and subproblems decomposed from the original problem. The simplification is achieved by using basic techniques such as first-order derivatives, second-order derivatives or roof-duality, and by using integrative techniques that combine the conclusions derived from the basic techniques. Computational experience on numerous problem classes shows the value of the proposed preprocessing techniques.

Palubeckis (2006) presents an iterated tabu search algorithm which uses a dedicated perturbation mechanism to enhance the high-quality solution obtained by the tabu search procedure. Each step of the perturbation constructs a candidate list of a limited size consisting of variables with largest 1-flip move gains with regard to this current solution, from which a variable is randomly selected and flipped to complement the value of this variable. The current solution is thus updated and the next perturbation step continues until the number of perturbed variables reaches the specified number. Comparisons with state-of-the-art algorithms disclose the competitiveness of the proposed algorithm in spite of its simplicity. 
Boros, Hammer, and Tavares (2007) present a local search scheme for solving UBQP. Starting from an initial solution, each iterative step constructs a candidate set from which a variable is picked and its value is changed to its complement, thus moving to the next solution. This iterative procedure repeats until the candidate set becomes empty. Based on the above scheme, they investigate five initialization methods, two candidate set construction methods and four variable selection methods, thus reaching up to 40 local search alternatives. Experiments on multiple benchmark instances indicate that the local search alternative combining the following methods achieves the best performance. The initial method assigns each variable with a fractional value equaling to the proportion of the sum of all the positive entries of the matrix in the sum of the absolute value of each entry of the matrix. The candidate set construction method constructs a candidate set consisting of variables that yield an improvement in the current solution by flipping its value regardless of whether or not it was already flipped in the previous iteration. The variable selection method selects from the candidate set the variable with the largest improvement to the current solution.

Glover, Lu, and Hao (2010) present a diversification-driven tabu search algorithm, which alternates between a basic tabu search procedure and a memory-based perturbation strategy guided by a long-term memory. Three memory structures are introduced in the perturbation strategy: (1) a flipping frequency vector to record the number of times a variable has been flipped from the initial iteration until the current iteration; (2) an elite set of solutions to record a certain number of best local optimal solutions; (3) a consistency vector to count the times each variable is assigned a given value in the set of elite solutions. Based on the memory information, the perturbation operator modifies an elite solution by favoring variables with low flipping frequency and high consistency to flip. Comparisons drawn with several algorithms proposed by Palubeckis $(2004,2006)$ disclose the superiority of this algorithm.

Lu, Glover, and Hao (2010) present a hybrid metaheuristic approach which has the following features. First, it combines a traditional uniform crossover operator with a diversification guided path relinking operator to guarantee the quality and diversity of an offspring solution. Second, it defines a new distance by reference to variable's importance and employs a quality-and-distance criterion to update the population. Finally, a tabu search procedure is responsible for intensified examination around the offspring solutions. 
Computational comparisons with best performing algorithms indicate the effectiveness of this hybrid algorithm.

$\mathrm{Lu}$, Hao, and Glover (2010) develop a hybrid genetic tabu search with multi-parent crossover to solve UBQP. The proposed algorithm jointly uses traditional uniform crossover and logic multi-parent combination operators to generate diversified offspring solutions. Computational experience is given showing the competitiveness of the proposed algorithm.

Cai, Wang, Yin, and Zhou (2011) present a memetic clonal selection algorithm with estimation of distribution algorithm (EDA) guided vaccination for solving UBQP. The proposed algorithm adopts EDA vaccination, fitness uniform selection scheme and adaptive tabu search to overcome the deficiencies of traditional clonal selection algorithm. Experimental comparisons indicate the tabu search algorithm enhances the performance of the clonal selection algorithm.

Shylo and Shylo (2011) develop a global equilibrium search which performs multiple temperature cycles. Each temperature cycle includes an initial solution generation phase and a tabu search phase. The method to generate an initial solution employs historical information to determine the probability that a variable receives the value 1 . The tabu search procedure requires that each admissible move leads to a solution with hamming distance to a reference set surpassing a distance threshold. Computational comparisons with several algorithms indicate the attractiveness of the proposed algorithm.

Hanafi, Rebai, and Vasquez (2013) devise five alternative DDT heuristics based on different representations of the BQO formulation. DDT1 to DDT4 respectively have standard, posiform, bi-form and negaform representations and DDT5 has a posiform representation combined with a one-pass mechanism. One obvious difference between the DDT alternatives proposed here and those proposed by Boros et al. (1989) and Glover et al. (2002) lies in the use of an r-flip local search procedure to improve solutions obtained by DDT constructions. Extensive tests on small, medium and large benchmark instances disclose that (1) DDT3 with the bi-form representation generally produces the best results for medium and large instances; (2) the $r$-flip local search contributes to significant improvement of the results of the proposed DDT methods with only a slight increase of time consumption. 
Wang, Zhou, and Yin (2011) present a tabu Hopfield neural network with an estimation of distribution algorithm (EDA). The cooperation between long term memory of EDA with the short term memory of tabu search prevents the network from becoming trapped in local optima. Computational testing indicates the superiority of the proposed algorithm compared to other Hopfield neural network based algorithms.

Lu, Glover, and Hao (2011) study neighborhood union and token-ring search methods within a tabu search algorithm. They focus on two neighborhoods, N1 consisting of 1-flip moves and N2 consisting of a chosen subset of 2-flip moves. The neighborhood union includes the strong neighborhood union that picks each move from both $\mathrm{N} 1$ and $\mathrm{N} 2$ and the selective neighborhood union that picks a move from $\mathrm{N} 1$ with probability $p$ and $\mathrm{N} 2$ with probability 1- $p$. The token ring search continuously performs move in N1 until no improvement is possible and then switches to perform move in N2 to continue the search. Computational comparisons reveal the superiority of the token ring search over the neighborhood union.

Wang, Lu, Glover and Hao (2012) present two path relinking algorithms, which are composed of a reference set construction method, a tabu search based improvement method, a reference set update method, a relinking method and a path solution selection method. The proposed algorithms differ from each other mainly on the way they generate the path, one employing a greedy strategy and the other employing a random strategy. Extensive computational experience and comparisons with several state-of-the-art algorithms highlight the attractiveness of the proposed algorithms in terms of both solution quality and computational efficiency.

Wang, Lu, Glover and Hao (2012) propose a simple GRASP-Tabu Search algorithm working with a single solution and an enhanced version by combining GRASP-Tabu Search algorithm with a population management strategy based on an elite reference set. In the basic version, the initial solution is constructed according to a greedy random construction heuristic. In the enhanced version, a new solution is reconstructed by first inheriting parts of the good assignments of one elite solution to form a partial solution and then completing the remaining parts as the basic version does. Experimental tests on a large range of both random and structured problem instances disclose that the proposed algorithms, in particular the enhanced version, yield very competitive outcomes. 
Wang, Lu, Glover, and Hao (2012) present a backbone guided tabu search algorithm which alternates between a basic tabu search procedure and a variable fixing/freeing phase based on identifying strongly determined variables. While the tabu search phase ensures the exploitation of a search space, the variable fixing (freeing) phase dynamically enlarges (reduces) the backbone of assigned values that launches the tabu search exploration. Experiments show that the proposed algorithm obtains highly competitive outcomes in comparison with the previous best known results from the literature. A direct comparison with the underlying tabu search procedure confirms the merit of incorporating backbone information.

As indicated in the papers of this section, our ability to efficiently solve large instances of UBQP by heuristic means has grown substantially in recent years. It is common now for authors to report computational experience on problems with 7,000-10,000 variables. Note that the set partitioning application discussed in section 2.3 by Lewis, et. al. (2008) reported computational experience on problems up to 15,000 variables.

\subsection{Key Theoretical Results}

By far the majority of the papers in the literature related to UBQP are primarily devoted to applications or various solution schemes, either exact or heuristic in nature. As a result, our priority in this paper has been to focus our survey on applications and solution methodologies. Many of the articles surveyed in section 3 above, however, contain a discussion of the theoretical results relevant to the method being put forth. That is, these papers are mainly about the method at hand but may also contain a discussion of underlying theory. As a result, we've not explicitly highlighted theoretical issues but rather left them to be discovered, as might be appropriate, as part of the articles on applications and solutions methods surveyed. Nonetheless, there are a few recent papers in the literature of particular note focused on theoretical issues pertaining to UBQP. It is these papers that we highlight here in this section.

Carraresi, Farinaccio and Malucello (1999) present an exact algorithm for testing the optimality of a given solution for a quadratic $0-1$ unconstrained problem. Their method, based on necessary and sufficient conditions introduced by Hirriart-Urruty for general convex problems, expands their earlier work (1995) which was an approximation scheme for testing solutions.

Beck and Teboulle (2000) characterize global optimal solutions for UBQP as well as discussing the relationship between optimal solutions to UBQP and the optimal solutions of its 
continuous relaxation. They derive a sufficient optimality condition which guarantees that a given feasible point is a global optimal for UBQP as well as a necessary global optimality condition.

Jeyakumar, Rubinov, and $\mathrm{Wu}$ (2007) examine the relationship between the global optimality of nonconvex constrained optimization and Lagrange multiplier conditions, establishing sufficient as well as necessary conditions for global optimality for general quadratic minimization problems with quadratic constraints. This analysis led, as a special case, to new sufficient and necessary global optimality conditions for UBQP that are sharper than those given earlier by Beck and Teboulle.

Xia (2009), by analyzing local sufficient optimality conditions, also extended the Beck and Teboulle results by developing tighter sufficient optimality conditions. In addition, without making the positive-semidefinite assumption, Xia examines the relationship between local/global minimizers of UBQP and the KKT points of the continuous relaxation, further extending previous results in the literature.

Gao and Ruan (2010) present a discussion of canonical duality theory, designed in general for a wide class of nonconvex/nonsmooth/discrete problems. The authors show how this duality theory can be adapted for the quadratic case with binary constraints. Conditions are given that allow instances of UBQP to be converted into smooth concave maximization dual problems over a closed convex feasible region without a duality gap. Finally, the relationship between canonical duality theory and semi-definite programming for UBQP is discussed.

Zheng, Sun, $\mathrm{Li}$, and $\mathrm{Xu}$ (2012) present new sufficient conditions for verifying zero duality gap in nonconvex constrained quadratic programs and then show how the results specialize for UBQP. In related work, Sun, Liu, and Gao (2012) investigate the duality gap between UBQP and its semi-definite programming relaxation. Making the connection between the duality gap and the cell enumerations of hyperplane arrangement in discrete geometry, estimates of the duality gap can be derived, yielding improved lower bounds for UBQP.

We note that there are several theoretical papers in the literature on the constrained version of UBQP that don't explicitly consider the pure UBQP model but are nonetheless relevant to our work in that UBQP is a special case of the constrained cases considered. Notably, Pinar (2004) gives a discussion of sufficient global optimality conditions for the problem of minimizing a quadratic function in binary variables subject to equality quadratic 
constraints. Lu, et. al., (2011) presents a discussion of KKT conditions and conic relaxations to develop sufficient conditions that generalize known positive semi-definiteness results for finding globally optimal solutions for the problem of minimizing a UBQP subject to inequality quadratic constraints. Finally, Li (2012) presents an extension of Pinar's global optimality conditions for the quadratic equality constrained case along with presenting conditions enabling global optimality to be assessed by checking the positive semi-definiteness of a related matrix.

\subsection{Summary \& Conclusions}

Interest in UBQP has grown substantially in recent years as researchers have discovered the remarkable ability of this simple model form to represent a wide variety of combinatorial problems along with its computational challenge, particularly as model sizes have increased. Due to its NP-hard nature, methods capable of producing exact solutions are limited to modest sized applications, giving way to modern heuristic methods for larger models. Even today, exact methods appear to be limited to a few hundred variables. In an effort to realize the application potential of UBQP as model size scales to higher levels, most research is focused on metaheuristic methods of one kind or another. The results are encouraging: Articles in the 80s were reporting on solving problems with 100-200 variables while more recent articles are reporting on problems with up to 15,000 variables. To a large extent, this growth in performance is due to advances in both algorithm design and computer hardware.

Successfully moving to the next order of magnitude in terms of model size will require creative schemes for handling very large Q matrices along with improved algorithmic methods. Various partitioning and multi-level methods hold particular promise here but the door is open for other innovations as well. Advances in computer performance, both in terms of storage and speed, can also be expected to lend a hand in allowing larger applications. Moreover, developments in the area of quantum computing, as illustrated by the work by Neven, Rose, and Macready (2008), represent emerging technologies with a potential for solving combinatorial problems as represented by UBQP. Future papers will reveal which of these research areas, or indeed, if some other approach, will contribute to facilitating solutions to UBQP as application size continues to scale upward. 


\subsection{References}

Alidaee B, Glover F, Kochenberger GA, Rego C (2005) A new modeling and solution approach for the number partitioning problem. Journal of Applied Mathematics \& Decision Sciences 2005 (2):113121. doi:10.1155/JAMDS.2005.113

Alidaee B, Kochenberger G, Lewis K, Lewis M, Wang H (2008) A new approach for modeling and solving set packing problems. European Journal of Operational Research 186 (2):504-512. doi:http://dx.doi.org/10.1016/j.ejor.2006.12.068

Alidaee B, Kochenberger GA, Ahmadian A (1994) 0-1 Quadratic programming approach for optimum solutions of two scheduling problems. International Journal of Systems Science 25 (2):401-408. doi:10.1080/00207729408928968

Alkhamis TM, Hasan M, Ahmed MA (1998) Simulated annealing for the unconstrained quadratic pseudoBoolean function. European Journal of Operational Research 108 (3):641-652. doi:http://dx.doi.org/10.1016/S0377-2217(97)00130-6

Amini MM, Alidaee B, Kochenberger GA (eds) (1999) A scatter search approach to unconstrained quadratic binary programs. New ideas in optimization. McGraw-Hill Ltd., UK,

Barahona $F$ (1986) A solvable case of quadratic 0-1 programming. Discrete Applied Mathematics 13 (1):23-26. doi:http://dx.doi.org/10.1016/0166-218X(86)90065-X

Barahona F, Grotschel M, Junger M, Reinelt G (1988) An Application of Combinatorial Optimization to Statistical. Operations Research 36 (3):493

Barahona F, Junger M, Reinelt G (1989) Experiments in Quadratic 0-1 Programming. Mathematical Programming 44:127-137

Beck A, Teboulle M (2000) Global Optimality Conditions for Quadratic Optimization Problems with Binary Constraints. SIAM J. of Optimization 11(1):179-188

Beasley JE (1998) Heuristic algorithms for the unconstrained binary quadratic programming problem. London, England

Billionnet A, Elloumi S (2007) Using a mixed integer quadratic programming solver for the unconstrained quadratic 0-1 problem. Mathematical Programming 109 (1):55-68

Billionnet A, Sutter A (1994) Minimization of a quadratic pseudo-Boolean function. European Journal of Operational Research 78 (1):106-115. doi:http://dx.doi.org/10.1016/0377-2217(94)90125-2

Bomze IM, Budinich M, Pardalos PM, Pelillo M (1999) The maximum clique problem. In: Handbook of combinatorial optimization. Springer, pp 1-74

Boros E, Hammer P, Sun X (1989) The DDT method for quadratic 0-1 minimization. RUTCOR Research Center, RRR:39-89

Boros E, Hammer PL (1991) The max-cut problem and quadratic 0-1 optimization polyhedral aspects, relaxations and bounds. Annals of Operations Research 33 (1-4):151-180

Boros E, Hammer PL (2002) Pseudo-Boolean optimization. Discrete Applied Mathematics 123 (1-3):155225. doi:http://dx.doi.org/10.1016/S0166-218x(01)00341-9

Boros E, Hammer PL, Tavares G (2006) Preprocessing of Unconstrained Quadratic Binary Optimization. R u t c or Research R e p ort, vol 13.

Boros E, Hammer PL, Tavares G (2007) Local search heuristics for Quadratic Unconstrained Binary Optimization (QUBO). Journal of Heuristics 13 (2):99-132

Cai Y, Wang J, Yin J, Zhou Y (2011) Memetic clonal selection algorithm with EDA vaccination for unconstrained binary quadratic programming problems. Expert Systems with Applications 38 (6):7817-7827. doi:http://dx.doi.org/10.1016/j.eswa.2010.12.124

Carraesi P, Malucelli F, Farinaccio F (1995) Testing Optimality for Quadratic 0-1 Unconstrained Problems. ZOR-Math. Meth. Operations Research 42:295-311. 
Carraesi P, Farinaccio F, Malucelli F (1999) Testing Optimality for Quadratic 0-1 Problems. Math. Programming 85: 407-421.

Carter MW (1984) The indefinite zero-one quadratic problem. Discrete Applied Mathematics 7 (1):23-44

De Simone C, Diehl M, Jünger M, Mutzel P, Reinelt G, Rinaldi G (1995) Exact ground states of Ising spin glasses: new experimental results with a branch-and-cut algorithm. Journal of Statistical Physics 80 (1-2):487-496

Douiri SM, Elbernouss S (2012) The Unconstrained Binary Quadratic Programming for the Sum Coloring Problem. Modern Applied Science 6 (9):26-33. doi:10.5539/mas.v6n9p26

Gao D, Ruan N (2010) Solutions to Quadratic Minimization Problems with Box and Integer Constraints. J. Global Optimization 47:463-484,. DOI 10.1007/s10898-009-9469-0

Glover F, Alidaee B, Rego C, Kochenberger G (2002) One-pass heuristics for large-scale unconstrained binary quadratic problems. European Journal of Operational Research 137 (2):272-287. doi:http://dx.doi.org/10.1016/S0377-2217(01)00209-0

Glover F, Kochenberger G, Alidaee B, Amini M (1999) Tabu search with critical event memory: an enhanced application for binary quadratic programs. In: Meta-Heuristics. Springer, pp 93-109

Glover F, Kochenberger GA, Alidaee B (1998) Adaptive memory tabu search for binary quadratic programs. Management Science 44 (3):336-345

Glover F, Lü Z, Hao J-K (2010) Diversification-driven tabu search for unconstrained binary quadratic problems. 4OR 8 (3):239-253

Gueye S, Michelon P (2009) A linearization framework for unconstrained quadratic (0-1) problems. $\begin{array}{llll}\text { Discrete } & \text { Applied } & \text { Mathematics } & 157\end{array}$ doi:http://dx.doi.org/10.1016/j.dam.2008.01.028

Gulati VP, Gupta SK, Mittal AK (1984) Unconstrained quadratic bivalent programming problem. European Journal of Operational Research 15 (1):121-125. doi:http://dx.doi.org/10.1016/03772217(84)90055-9

Hammer P, Shlifer E (1971) Applications of pseudo-Boolean methods to economic problems. Theor Decis 1 (3):296-308. doi:10.1007/BF00139572

Hammer PL, Rudeanu S (1968) Boolean methods in operations research and related areas, vol 5. Springer-Verlag Berlin,

Hanafi S, Rebai AR, Vasquez M (2013) Several versions of the devour digest tidy-up heuristic for unconstrained binary quadratic problems. Journal of Heuristics 19 (4):645-677

Hansen P (1979) Methods of Nonlinear 0-1 Programming. In: P.L. Hammer EL, Korte BH (eds) Annals of Discrete Mathematics, vol Volume 5. Elsevier, pp 53-70. doi:http://dx.doi.org/10.1016/S01675060(08)70343-1

Hansen P, Jaumard B (1990) Algorithms for the maximum satisfiability problem. Computing 44 (4):279303

Hansen P, Jaumard B, Mathon V (1993) State-of-the-Art Survey-Constrained Nonlinear 0-1 Programming. ORSA Journal on Computing 5 (2):97-119

Hansen P, Jaumard B, Meyer C (2000) Exact sequential algorithms for additive clustering. Montréal: Groupe d'études et de recherche en analyse des décisions,

Helmberg C, Rendl F (1998) Solving quadratic $(0,1)$-problems by semidefinite programs and cutting planes. Mathematical Programming, Series B 82 (3):291-315

Huang H-X, Pardalos PM, Prokopyev OA (2006) Lower bound improvement and forcing rule for quadratic binary programming. Computational Optimization and Applications 33 (2-3):187-208

lasemidis L, Pardalos P, Sackellares J, Shiau D-S (2001) Quadratic binary programming and dynamical system approach to determine the predictability of epileptic seizures. Journal of Combinatorial Optimization $5(1): 9-26$ 
Jeyakumar V, Rubinov A. M, Wu Z. Y (2007) Non-convex Quadratic Minimization Problems with quadratic Constraints: Global Optimality Conditions. Math Programming, Series A 110:521-541 DOI 10.1007/s10107-006-0012-5

Kalantari B, Bagchi A (1990) An algorithm for quadratic zero-one programs. Naval Research Logistics (NRL) 37 (4):527-538.

Katayama K, Narihisa H (2001) Performance of simulated annealing-based heuristic for the unconstrained binary quadratic programming problem. European Journal of Operational Research 134 (1):103-119. doi:http://dx.doi.org/10.1016/S0377-2217(00)00242-3

Katayama K, Tani M, Narihisa $\mathrm{H}$ Solving large binary quadratic programming problems by effective genetic local search algorithm. In: Proc. 2000 Genetic and Evolutionary Computation Conference, 2000. pp 643-650

Kernighan B, Lin S (1970) An eflicient heuristic procedure for partitioning graphs. Bell system technical journal

Kochenberger G, Alidaee B, Glover F, Wang H (2007) An effective modeling and solution approach for the generalized independent set problem. Optimization Letters 1 (1):111-117

Kochenberger G, Glover F, Alidaee B, Lewis K (2005) Using the unconstrained quadratic program to model and solve Max 2-SAT problems. International Journal of Operational Research 1 (1):89100

Kochenberger G, Glover F, Alidaee B, Rego C (2005) An Unconstrained Quadratic Binary Programming Approach to the Vertex Coloring Problem. Annals of Operations Research 139 (1-4):229-241. doi:10.1007/s10479-005-3449-7

Kochenberger G, Glover F, Alidaee B, Wang H (2005) Clustering of microarray data via clique partitioning. Journal of Combinatorial Optimization 10 (1):77-92

Kochenberger GA, Hao J-K, Lü Z, Wang H, Glover F (2013) Solving large scale max cut problems via tabu search. Journal of Heuristics 19 (4):565-571

Krarup J, Pruzan P (1978) Computer-aided layout design. In: Balinski ML, Lemarechal C (eds) Mathematical Programming in Use, vol 9. Mathematical Programming Studies. Springer Berlin Heidelberg, pp 75-94. doi:10.1007/BFb0120827

Laughhunn D (1970) Quadratic binary programming with application to capital-budgeting problems. Operations Research 18 (3):454-461

Lewis $M$, Alidaee B, Glover F, Kochenberger $G$ (2009) A note on $x Q x$ as a modelling and solution framework for the Linear Ordering Problem. International Journal of Operational Research 5 (2):152-162

Lewis $M$, Alidaee $B$, Kochenberger $G$ (2005) Using $x Q x$ to model and solve the uncapacitated task allocation problem. Operations Research Letters 33 (2):176-182. doi:10.1016/j.orl.2004.04.014

Lewis M, Kochenberger G, Alidaee B (2008) A new modeling and solution approach for the setpartitioning problem. Computers \& Operations Research 35 (3):807-813. doi:10.1016/j.cor.2006.04.002

Lewis M, Kochenberger G, Wang H, Glover F (2013) Exact Solutions to Generalized Vertex Covering Problems: A Comparison of Two Models. working paper

Li D, Sun XL, Liu CL (2012) An exact solution method for unconstrained quadratic 01 programming: a geometric approach. Journal of Global Optimization 52 (4):797-829

Li G (2012) Global Quadratic Minimization over Bivalent Constraints: Necessary and Sufficient Global Optimality Condition, J. Optimization Theory, 52:710-726 DOI 10.1007/s10957-011-9930-3

Lodi A, Allemand K, Liebling TM (1999) An evolutionary heuristic for quadratic 0-1 programming. $\begin{array}{lllll}\text { European Journal of Operational Research } 119 & \text { (3):662-670. }\end{array}$ doi:http://dx.doi.org/10.1016/S0377-2217(98)00359-2 
Lu C, Fang A, Jin Q, Wang Z, Xing W (2011) KKT Solution and Conic Relaxation for Solving Quadratically Constrained Quadratic Programming Problems. SIAM J. Optimization 21(4):1475-1490

Lü Z, Glover F, Hao J-K (2010) A hybrid metaheuristic approach to solving the UBQP problem. European Journal of Operational Research 207 (3):1254-1262. doi:10.1016/j.ejor.2010.06.039

Lü Z, Hao J-K, Glover F (2010) A study of memetic search with multi-parent combination for UBQP. In: Evolutionary Computation in Combinatorial Optimization. Springer, pp 154-165

Lü Z, Hao J-K, Glover F (2011) Neighborhood analysis: a case study on curriculum-based course timetabling. Journal of Heuristics 17 (2):97-118. doi:10.1007/s10732-010-9128-0

Mahdavi Pajouh F, Balasundaram B, Prokopyev OA (2013) On characterization of maximal independent sets via quadratic optimization. Journal of Heuristics 19 (4):629-644

Mauri GR, Lorena LAN (2011) Lagrangean decompositions for the unconstrained binary quadratic programming problem. International Transactions in Operational Research 18 (2):257-270. doi:10.1111/j.1475-3995.2009.00743.x

Mauri GR, Lorena LAN (2012) A column generation approach for the unconstrained binary quadratic programming problem. European Journal of Operational Research 217 (1):69-74. doi:http://dx.doi.org/10.1016/j.ejor.2011.09.016

Mauri GR, Lorena LAN (2012) Improving a Lagrangian decomposition for the unconstrained binary quadratic programming problem. Computers \& Operations Research 39 (7):1577-1581. doi:http://dx.doi.org/10.1016/j.cor.2011.09.008

Merz P, Freisleben B Genetic algorithms for binary quadratic programming. In: Proceedings of the genetic and evolutionary computation conference, 1999. Citeseer, pp 417-424

Merz P, Freisleben B (2002) Greedy and local search heuristics for unconstrained binary quadratic programming. Journal of Heuristics 8 (2):197-213

Merz P, Katayama K (2004) Memetic algorithms for the unconstrained binary quadratic programming problem. Biosystems 78 (1-3):99-118. doi:http://dx.doi.org/10.1016/j.biosystems.2004.08.002

Neven H, Rose G, Macready WG (2008) Image recognition with an adiabatic quantum computer I. Mapping to quadratic unconstrained binary optimization.

Oosten M, Rutten J, Spieksma F (2001) The Clique partitioning problem: Facets and patching facets. Networks 38 (4):209-226

Palubeckis G (1995) A heuristic-based branch and bound algorithm for unconstrained quadratic zero-one programming. Computing 54 (4):283-301. doi:10.1007/BF02238228

Palubeckis G (2004) Multistart Tabu Search Strategies for the Unconstrained Binary Quadratic Optimization Problem. Annals of Operations Research 131 (1-4):259-282. doi:10.1023/B:ANOR.0000039522.58036.68

Palubeckis G (2006) Iterated Tabu Search for the Unconstrained Binary Quadratic Optimization Problem. Informatica 17 (2):279-296

Palubeckis G, Tomkevicius A (2002) \{GRASP\} implementations for the unconstrained binary quadratic optimization problem. Information Technology and Control 24:14-20

Pan S, Tan T, Jiang Y (2008) A global continuation algorithm for solving binary quadratic programming problems. Computational Optimization \& Applications 41 (3):349-362. doi:10.1007/s10589-0079110-4

Pardalos PM, Jha S (1991) Graph separation techniques for quadratic zero-one programming. Computers \& Mathematics with Applications 21 (6-7):107-113. doi:http://dx.doi.org/10.1016/08981221(91)90165-Z

Pardalos PM, Jha S (1992) Complexity of uniqueness and local search in quadratic 0-1 programming. Operations Research Letters 11 (2):119-123. doi:http://dx.doi.org/10.1016/01676377(92)90043-3 
Pardalos PM, Prokopyev OA, Busygin S (2006) Continuous approaches for solving discrete optimization problems. In: Handbook on modelling for discrete optimization. Springer, pp 39-60

Pardalos PM, Rodgers GP (1990) Computational aspects of a branch and bound algorithm for quadratic zero-one programming. Computing 45 (2):131-144

Pardalos PM, Rodgers GP (1990) Parallel branch and bound algorithms for quadratic zero-one programs on the hypercube architecture. Annals of Operations Research 22 (1-4):271-292

Pardalos PM, Rodgers GP (1992) A branch and bound algorithm for the maximum clique problem. Computers \& Operations Research 19 (5):363-375. doi:http://dx.doi.org/10.1016/03050548(92)90067-F

Pardalos PM, Xue J (1994) The maximum clique problem. Journal of global Optimization 4 (3):301-328

Pham Dinh T, Nguyen Canh N, Le Thi HA (2010) An efficient combined DCA and B\&B using DC/SDP relaxation for globally solving binary quadratic programs. Journal of Global Optimization 48 (4):595-632

Picard J-C (1976) Maximal Closure of a Graph and Applications to Combinatorial Problems. Management Science 22 (11):1268-1272. doi:10.2307/2630227

Pinar M. C (2004) Sufficient Global Optimality Conditions for Bivalent Quadratic Optimization. Journal of Optimization Theory and Applications 122(2):433-440

Rao MR (1971) Cluster Analysis and Mathematical Programming. Journal of the American Statistical Association 66 (335):622-626. doi:10.1080/01621459.1971.10482319

Rhys J (1970) A selection problem of shared fixed costs and network flows. Management Science 17 (3):200-207

Shylo V, Shylo O (2011) Systems Analysis Solving unconstrained binary quadratic programming problem by global equilibrium search. Cybernetics \& Systems Analysis 47 (6):889-897. doi:10.1007/s10559-011-9368-5

Sun X. L., Liu C. L, Li D, Gao, J. J (2012) On Duality Gap in binary Quadratic Programming. J. Global Optimization 53:255-269 DOI 10.1007/s10898-011-9683-4

Wang F, Xu Z (2013) Metaheuristics for robust graph coloring. Journal of Heuristics 19 (4):529-548

Wang H, Alidaee B, Glover F, Kochenberger G (2006) Solving group technology problems via clique partitioning. International Journal of Flexible Manufacturing Systems 18 (2):77-77

Wang J, Zhou Y, Yin J (2011) Combining tabu Hopfield network and estimation of distribution for unconstrained binary quadratic programming problem. Expert Systems with Applications 38 (12):14870-14881. doi:http://dx.doi.org/10.1016/j.eswa.2011.05.060

Wang Y, Lü Z, Glover F, Hao J-K (2012) A multilevel algorithm for large unconstrained binary quadratic optimization. In: Integration of $\mathrm{Al}$ and $\mathrm{OR}$ Techniques in Contraint Programming for Combinatorial Optimzation Problems. Springer, pp 395-408

Wang Y, Lü Z, Glover F, Hao J-K (2012) Path relinking for unconstrained binary quadratic programming. European Journal of Operational Research 223 (3):595-604. doi:http://dx.doi.org/10.1016/j.ejor.2012.07.012

Wang Y, Lü Z, Glover F, Hao J-K (2012) Probabilistic GRASP-tabu search algorithms for the UBQP problem. Computers \& Operations Research

Williams HP (1985) Model Building in Linear and Integer Programming. In: Schittkowski K (ed) Computational Mathematical Programming, vol 15. NATO ASI Series. Springer Berlin Heidelberg, pp 25-53. doi:10.1007/978-3-642-82450-0_2

Witzgall C (1975) Mathematical methods of site selection for Electronic Message Systems (EMS). NBS Internal report. NBS

Xia Y (2009) New Optimality Conditions for Quadratic Optimization Problems with Binary Constraints. Optimization Letters 3:253-263 DOI 10.1007/s11590-008-0105-6 
Zheng X.J, Sun X. L, Li D, Xu Y.F (2012) On Zero Duality Gap in Nonconvex Quadratic Programming Problems, J. Global Optimization 52:229-242 DOI 10.1007/s10898-011-9660-y 